\section{PRÁTICAS CORPORAIS E ATIVIDADES FÍSICAS NA ATENÇÃO BÁSICA DO SISTEMA ÚNICO DE SAÚDE: IR ALÉM DA PREVENÇÃO DAS DOENÇAS CRÔNICAS NÃO TRANSMISSÍVEIS É NECESSÁRIO}

\author{
BODY PRACTICES AND PHYSICAL ACTIVITIES IN PRIMARY HEALTH CARE IN \\ BRAZIL'S UNIFIED HEALTH SYSTEM: THE NEED TO GO BEYOND CHRONIC \\ DISEASE PREVENTION
}

\section{PRÁCTICAS CORPORALES Y ACTIVIDAD FÍSICA EN LA ATENCIÓN PRIMARIA DEL SISTEMA ÚNICO DE SALUD: IR MÁS ALLÁ DE LA PREVENCIÓN DE LAS ENFERMEDADES CRÓNICAS NO TRANSMISIBLES ES NECESARIO}

\section{Fabio Fortunato Brasil de Carvalho*}

Keywords Health promotion. Primary Health Care. Unified Health System.

Exercise.

\section{Palavras-chave} Promoção da saúde. saúde.

Sistema Único

de Saúde.

Exercício.
Resumo: 0 objetivo do ensaio é defender que as práticas corporais e atividades físicas que são ofertadas na Atenção Básica do Sistema Único de Saúde não se resumam apenas a atividades de prevenção das doenças crônicas não transmissíveis, sem desconsiderar a importância de tais ações. São reflexões e ideias que poderão contribuir para que trabalhadores da Atenção Básica possam auxiliar sujeitos e coletividades a agirem em favor de sua saúde através daquelas práticas. Assim, as práticas corporais e atividades físicas são compreendidas num sentido mais amplo, destacando-se 0 encontro, a convivência, a formação e fortalecimento de grupos sociais nos territórios ao criar e/ou aumentar o vínculo entre os sujeitos e destes com os trabalhadores e serviços de saúde.

Abstract: The purpose of this essay is to argue that body practices and physical activities offered by Primary Health Care under the Unified Health System are not restricted to activities for chronic disease prevention - without ignoring the importance of such actions. These are reflections and ideas that may contribute for Primary Health Care workers to help individuals and communities to improve their health through those practices. Thus, body practices and physical activity are understood in a broader sense, especially as encounters, coexistence, education, as well as strengthening of social groups in the territories by creating and/or enhancing bonds among subjects and between them and health workers and services.

Resumen: El propósito de este ensayo es defender que las prácticas corporales y las actividades físicas que se ofrecen en la atención primaria del Sistema Único de Salud no se resuman solamente a actividades de prevención de enfermedades crónicas no transmisibles, sin desmerecer la importancia de este tipo de acciones. Son reflexiones e ideas que podrían contribuir para que trabajadores de la Atención Primaria puedan auxiliar a individuos y colectivos para que actúen en favor de su salud a través de esas prácticas. Así, las prácticas corporales y actividades físicas son entendidas en un sentido más amplio, donde se destacan el encuentro, la convivencia, la formación y el fortalecimiento de grupos sociales en los territorios al crear y/o aumentar el vínculo entre los sujetos y entre éstos y trabajadores y servicios de salud.
*Escola Nacional de Saúde Pública Sergio Arouca. Fundação Oswaldo Cruz. Rio de Janeiro, RJ, Brasil. E-mail: fabiofbcarvalho@gmail.com

Recebido em: 31-08-2015 Aprovado em: 10-10-2015 (c) (1) (8) Licence Salud.

Ejercicio. 


\section{INTRODUÇÃO}

Atualmente as práticas corporais e atividades físicas (PC e AF) encontram grande aceitação no Sistema Único de Saúde (SUS) a partir da crença de que um "estilo de vida ativo" necessariamente estaria ligado a melhores condições de saúde e de análises críticas sobre esta perspectiva (ANDRADE, 2011, BAGRICHEVSKY; ESTEVÃO, 2008, CARVALHO, 2009, MALTA et al., 2008). Em comum, há a concordância de que elas podem contribuir para a saúde individual e coletiva.

É possível destacar duas principais interpretações a partir da relação das PC e AF com a saúde: como fator de proteção para as Doenças Crônicas Não Transmissíveis (DCNTs), rivalizando com os fatores de risco, baseada em grande parte na "epidemia de números" que fomenta a "pedagogia do terror" que permeia as políticas de promoção da $\mathrm{AF}^{1}$ (FRAGA, 2006). Esta "epidemia" se refere aos dados estatísticos que prometem eficácia às AF no combate ao desenvolvimento das DCNTs. Palma e Vilaça (2010) destacam os limites teórico-metodológicos dos estudos epidemiológicos que se propõem a medir o sedentarismo ou a inatividade física, em especial pela imprecisão proporcionada por incongruências entre os valores das distintas medidas de sedentarismo ou inatividade física em diferentes estudos.

A análise de um dos principais programas de promoção das AF permitiu a identificação do predomínio do caráter utilitário e individualista no processo de promoção da "vida ativa", com isso a construção de laços de sociabilidade e a ludicidade perdem importância já que o valor maior atribuído às AF está cada vez mais vinculado aos seus propalados efeitos orgânicos (FRAGA, 2006).

A outra interpretação se refere a uma perspectiva que extrapola apenas a doença e 0 aspecto biológico do corpo e da saúde na qual o destaque é para o encontro e a formação de vínculos entre os sujeitos, criando e fortalecendo grupos sociais.

A partir da Estratégia Global sobre Alimentação Saudável, Atividade Física e Saúde lançada pela Organização Mundial da Saúde (OMS) em 2003, com seu foco nas DCNTs, e da assunção pelo Ministério da Saúde (MS) desta estratégia, as AF enquanto fator de proteção torna-se uma das principais atividades de Promoção da Saúde (CRUZ, 2010). A Política Nacional de Promoção da Saúde (PNPS) confirmou o reconhecimento das PC e AF, uma das ações específicas, no SUS (MALTA et al., 2014). Ressalta-se que a definição das ações específicas desta política foi um dos maiores pontos de conflito (CRUZ, 2010), justamente porque deu margem para a realização de ações eminentemente preventivas.

Atualmente as PC e AF estão amplamente difundidas na Atenção Básica (AB) no SUS, inicialmente a partir do incentivo técnico e financeiro dado pelo MS para fortalecer a PNPS que contou com 1.079 municípios com projetos entre 2005 e 2010 (MALTA et al., 2014), entre outras iniciativas, e posteriormente com o Programa Academia da Saúde², que já conta com mais de quatro mil polos em mais de dois mil e oitocentos municípios brasileiros (BRASIL, 2014a).

Este ensaio tem o objetivo de defender que as $\mathrm{PC}$ e $\mathrm{AF}$ na $\mathrm{AB}$ não se resumam apenas a atividades de prevenção das DCNTs, sem desconsiderar a importância de tais ações. São

1 Quando se tratar de citações, a opção dos autores será respeitada e por isso os termos/conceitos atividade física ou práticas corporais aparecerão diferenciados. Essa diferenciação será objeto de problematização no presente texto.

20 objetivo principal do programa é contribuir para a promoção da saúde e produção do cuidado e de modos de vida saudáveis da população a partir da implantação de polos com infraestrutura e profissionais qualificados. As práticas corporais e atividades físicas são o eixo mais 
reflexões e ideias que poderão contribuir para que trabalhadores da $A B$ possam auxiliar sujeitos e coletividades a agirem em favor de sua saúde.

Para isso apontaremos a polarização entre os termos/conceitos: práticas corporais e 0 de atividade física. Para alguns autores eles são indissociáveis e antagônicos, já para outros há a possibilidade de complementação. Abordaremos ainda as PC e AF na atuação profissional na $A B$ e posteriormente defenderemos a necessidade delas não se resumirem à prevenção das DCNTs, como hegemonicamente têm sido difundidas, assim como faremos algumas considerações.

\section{METODOLOGIA}

Este ensaio consiste em uma revisão de literatura não sistematizada mediante a busca de informações em periódicos, livros e documentos oficiais sobre o tema que permitem situar os conhecimentos sobre a questão tratada no ensaio, qual seja, a relação das PC e AF na $A B$ com a saúde, sem ter o objetivo de esgotar as possibilidades que o tema oferece.

\section{AS PRÁTICAS CORPORAIS E ATIVIDADES FÍSICAS: CONCEITOS, POSSÍVEIS SENTIDOS E ATUAÇÃO DOS PROFISSIONAIS E TRABALHADORES NA ATENÇÃO BÁSICA}

Situar o debate entre os termos/conceitos práticas corporais e atividades físicas é importante para ir além da prevenção das DCNTs porque hegemonicamente apenas os benefícios biológicos têm sido difundidos e, a priori, apenas as AF, em uma visão restrita que será abordada, estariam relacionadas a tais benefícios.

Uma definição pode não restringir ou impedir que existam diversos sentidos para um conceito, isso é relevante ao tratar os termos/conceitos práticas corporais e atividade física, já que há, na literatura, em especial na que aproxima a Educação Física das Ciências Sociais e da Saúde Coletiva, um importante embate de forças.

Lazzarotti Filho et al. (2010), ao buscar identificar os significados/sentidos com os quais o termo PC vem sendo utilizado na literatura acadêmica, afirmam que ele surge nas publicações na década de 1990 remetendo, entre outras coisas, à ampliação conceitual com elementos das Ciências Humanas e Sociais.

Alguns autores (DAMICO; KNUTH, 2014, FRAGA et al., 2013, MARTINEZ et al., 2013) afirmam que o debate ganha ainda mais visibilidade a partir de publicações do MS, em especial a PNPS em 2006, por estarem presentes em marcos legais do SUS. O debate enunciado parte da ideia comum de que são importantes para a saúde, contudo, a concepção que embasa esta ideia difere consideravelmente.

A AF é o "movimento corporal que produz gasto de energia acima dos níveis de repouso" (BRASIL, GTPS, 2012a, p. 17) ${ }^{3}$, já as PC são as "[...] expressões individuais ou coletivas do movimento corporal, advindo do conhecimento e da experiência em torno do jogo, da dança, do esporte, da luta, da ginástica, construídas de modo sistemático (na escola) ou não sistemático (tempo livre/lazer)" ((BRASIL, GTPS, 2012a, p. 28). Nesta mesma publicação,

3 A opção por utilizar o Glossário Temático: Promoção da Saúde (Brasil, 2012) como fonte das definições é intencional, ele é muito utilizado para embasar o debate e o embate trazidos neste ensaio. 
ainda há a complementação deste último conceito que traz a dimensão dos significados que as pessoas atribuem às manifestações da cultura corporal de determinado grupo, contemplando as vivências lúdicas e culturais.

Para Damico e Knuth (2014) a diferenciação desses termos/conceitos produz sentidos de poder e saber extremamente conflitantes e duais, segundo Yara Maria de Carvalho (2007), o conceito de AF homogeneizaria o coletivo e padronizaria o corpo. Ferla et al. (2013), ao se referirem a ela, tratam de propostas sistematizadas de movimento que consideram apenas o biofisiológico dos indivíduos. Os últimos autores afirmam que o termo/conceito PC estaria ligado aos processos de subjetivação, às manifestações culturais, entre outros fatores.

Assim, há dois extremos: havendo a quantificação energética, necessariamente ocorre a destituição de sentidos e significados do movimento corporal para os sujeitos, ou partindo/ considerando a subjetividade e cultura destes, sendo lúdico, o movimento não necessitaria de energia para ocorrer.

Contudo, é possível encontrar na literatura alguns autores que buscam, de alguma forma, aproximar os conceitos. Fabio Fortunato Brasil de Carvalho (2012a) faz a apropriação do termo/conceito AF para denominar um campo geral que englobe o desporto, o lazer, a aptidão física, as PC e exercícios físicos a partir da crença da necessidade de sua compreensão num sentido amplo, o que extrapolaria a simples quantificação energética. Para este autor, seria possível que as PC fizessem parte do termo/conceito AF, num sentido ampliado. Também se encontra na literatura a proposta de uma sistematização das PC na qual o exercício físico ${ }^{4}$ é incluído (BRASIL, 2013).

Damico e Knuth (2014), ao se referirem ao saber epidemiológico, afirmam que é possível que o entendimento das AF não seja em um sentido unicamente biológico. Estes autores destacam que o debate que envolve tais conceitos ainda é inoperante no cotidiano dos serviços de saúde, sendo mais robusto no enfrentamento epistemológico.

Reiteramos então a afirmativa inicial de que podem ser atribuídos distintos sentidos para um conceito, a partir daí tanto é possível compreender o conceito de AF num sentido mais amplo, que não considere apenas a energia despendida para a realização do movimento corporal e, com isso, exclusivamente o aspecto biológico, assim como é possível compreender o conceito de PC que não exclua este mesmo aspecto.

Ainda não há um consenso que relacione apenas um dos termos/conceitos ao sentido amplo apresentado aqui, que una os aspectos subjetivo, cultural, histórico, social e o biológico. Optamos pela utilização do termo práticas corporais e atividades físicas a partir da defesa da compreensão dele de forma ampliada, sem desconsiderar os aspectos citados e sem hierarquizá-los.

Para ampliar o escopo das PC e AF na AB é necessário abordá-las a partir da atuação profissional, assim neste momento abordaremos a questão já que para ir além da prevenção das DCNTs é necessário considerar as especificidades das profissões, buscando a superação da fragmentação das ações de saúde pelo recorte da atuação profissional.

Reconhecemos as especificidades da atuação dos profissionais e trabalhadores da $A B$, em especial os técnicos e de ensino superior que têm formação específica e inscrição em 
conselhos profissionais. Contudo, há uma intercessão grande nos conhecimentos, habilidades e atitudes relacionadas às práticas de saúde, por exemplo: uma atividade de conscientização corporal, em especial a que propõe a unificação do corpo que possui sistemas e órgãos com o corpo emotivo e que expressa a forma de interação dos sujeitos com o mundo, baseado na compreensão de que é o mesmo corpo, é restrita à atuação de um ou outro profissional?

O fortalecimento da produção do cuidado de forma multiprofissional e transdisciplinar, uma necessidade para a efetivação da integralidade, necessita que limites demarcados pela atuação profissional tornem-se menos rígidos, através da articulação dos conhecimentos e enriquecimento de competências dos envolvidos, o que fortalece a capacidade de cuidado de toda a equipe (BRASIL, 2013).

Além da formação profissional, há uma formação complementar que ocorre ao longo da vida do trabalhador da saúde, as PC e AF orientais como o Lian Gong ou o Tai-Chi-Chuan, amplamente ofertadas na $A B$, são exemplos. Qual é o profissional que pode atuar com elas?

Propomos então a reflexão: como os trabalhadores da saúde se apropriam dos princípios e diretrizes da $A B$, do SUS, assim como de algumas ferramentas conceituais e operacionais, por exemplo, a integralidade ${ }^{5}$, atributos das práticas dos trabalhadores e profissionais de saúde e atributos da organização dos serviços (MATTOS, 2006). A integralidade aqui se refere aos arranjos planejados e operacionalizados pelos serviços e equipes de saúde da $A B$ a fim de que os usuários tenham acesso a respostas para suas necessidades de saúde, o que inclui as redes de atenção e a de produção social da saúde (intersetorialidade) (TURA et al., 2014).

Assim, como o profissional lida com a superação da abordagem fragmentária da condição de saúde dos sujeitos? Como se enquadra na lógica da organização dos serviços de saúde para que estes sejam cada vez mais capazes de influir positivamente na vida e saúde dos sujeitos e coletividades? Como ferramenta conceitual e operacional, o exemplo é a intersetorialidade, compreendida como a busca e a promoção de mudanças nas condições de vida e de saúde da população mediante a visão integrada dos complexos problemas sociais da atualidade, 0 que requer a inclusão de vários atores sociais e seus olhares, além da interação das diversas políticas sociais através de medidas multidisciplinares (CARVALHO, 2012b). É possível que apenas um determinado profissional possibilite a promoção das mudanças citadas? Como as competências específicas são articuladas com as de outros trabalhadores e profissionais? Isso é conseguido na formação e na inscrição do respectivo conselho profissional?

Esses questionamentos têm múltiplas respostas, o que queremos é problematizar a avaliação da atuação na $A B$ com as PC e AF exclusivamente a partir da formação e inscrição num determinado conselho profissional. Sem dúvidas, esses itens devem ser considerados, mas juntamente com outras competências, para que se aumente a possibilidade de ir além da prevenção das DCNTs.

Não está se propondo que qualquer profissional atue indistintamente com qualquer tipo/ modalidade das PC e AF, é inegável que determinados profissionais têm maior aproximação com a temática, contudo a demarcação das diferentes possibilidades de atuação com elas não pode permitir que a disputa por um campo de poder inviabilize a oferta das PC e AF para a população usuária da $\mathrm{AB}$. Tal disputa refere-se à defesa da exclusividade de determinados profissionais na oferta das PC e AF independentemente dos seus objetivos e contexto.

5 Diversas publicações avançam na problematização e compreensão deste princípio, a forma relativamente simples como é trazido neste ensaio tem o objetivo de compor a reflexão sobre a atuação profissional com as PC e AF. 
O estabelecimento de diálogo entre as instituições formadoras, as de regulação do exercício profissional e a gestão do SUS, é necessário para a construção de possibilidades com o objetivo maior de oferecer diferentes opções de PC e AF na AB, por exemplo, a pactuação de quais modalidades podem ser ofertadas por diferentes trabalhadores e profissionais.

\section{PARA IR ALÉM DA PREVENÇÃO DAS DOENÇAS CRÔNICAS NÃO TRANSMISSíVEIS NA ATENÇÃO BÁSICA}

De forma sucinta, abordaremos as características e diretrizes da AB. Trata-se de um "conjunto de ações de saúde, no âmbito individual e coletivo, que abrange a promoção e a proteção da saúde, a prevenção de agravos, o diagnóstico, o tratamento, a reabilitação, a redução de danos e a manutenção da saúde" (BRASIL, PNAB, 2012b, p. 19). A partir daí é possível afirmar a amplitude, complexidade e importância de seus serviços e ações de saúde (SILVA; CARVALHO, 2014).

Para impactar na situação de saúde e autonomia de sujeitos e coletividades e nos determinantes e condicionantes da saúde, o objetivo da AB é desenvolver uma atenção integral, assumindo responsabilidade sanitária sobre uma população e território definidos, levando em conta a dinamicidade deste território e utilizando o trabalho em equipe (BRASIL, PNAB, 2012b), logo transdisciplinar e multiprofissional. Busca considerar sujeitos, coletividades e o território em suas singularidades e características sociais e culturais, contribuindo para consolidar os princípios do SUS. A AB deve ser a principal porta de entrada e o contato preferencial de sujeitos e coletividades com os serviços de saúde.

Atualmente, as PC e AF encontram grande aceitação no imaginário social e coletivo, já que ocorre uma massificação destas como um importante componente para a saúde individual e coletiva. Tal massificação pode ser vista a partir do apelo midiático, dos trabalhadores da saúde e até mesmo na Lei Orgânica da Saúde 6 .

Segundo pesquisas do MS, a disponibilidade de espaços públicos para sua realização eleva a frequência destas práticas nessas localidades (BRASIL, 2011). Em relação a essa temática, ainda há muitas crenças e mitos, eles são (re)criados e renovados também pela massificação citada, por exemplo: no envelhecimento, até pouco tempo, se acreditava que determinadas modalidades não eram adequadas, a partir da compreensão da velhice como sinônimo de fragilidade, assim o treinamento resistido (musculação ou ginástica localizada) era contraindicado e a hidroginástica seria mais recomendada. Ou que a natação seria a melhor opção para o adolescente crescer.

As PC e AF englobam uma diversidade de ações e podem trazer benefícios que estão amplamente evidenciados na literatura, destacam-se os:

- socioafetivos, como a maior inserção e interação na comunidade, o aumento da autoestima, a diminuição nos níveis de ansiedade e depressão;

- biológicos, como a prevenção das DCNT e seus fatores de risco, a prevenção e combate da osteoporose através do aumento e/ou manutenção da densidade mineral óssea, a diminuição ou cessação de dores crônicas;

- cognitivos, como o aprimoramento da memória. 
São exemplos de PC e AF: jogos cooperativos e competitivos, brincadeiras, esportes, exercícios resistidos, outras modalidades de ginásticas, atividades recreativas, lutas, danças, atividades de aventura, movimentos corporais que levem o sujeito (re)conhecer seu corpo e suas partes, que trabalhem a coordenação motora e equilíbrio, agilidade, relacionadas à postura, alongamentos e relaxamentos, exercícios respiratórios, caminhada e corrida, entre outros.

É relevante destacar que a realização das PC e AF está relacionada a um processo complexo, não dependente apenas da vontade dos sujeitos, pois há muitos fatores intervenientes, a saber: o papel dos meios de comunicação, a cultura local, aconselhamento de profissionais de saúde, apoio de familiares (em especial o cônjuge), disponibilidade de tempo, conhecimento e acesso a instalações e espaços adequados, condições socioeconômicas, experiências anteriores, entre outros (FERREIRA; NAJAR, 2005, PALMA, 2000). Os trabalhadores da AB devem considerar esses fatores, buscando minimizar abordagens simplistas sobre a questão, em especial, aquelas que culpabilizam o usuário pela não adesão a elas.

Uma barreira de acesso comum é a obrigatoriedade de uma avaliação física ou funcional para a realização, na AB, das PC e AF. Há variadas formas de avaliar as condições de saúde de sujeitos e coletividades: através de anamnese baseada em uma conversa informal em que se busque conhecer o histórico de cada sujeito, pela observação dos participantes das atividades, através de exames complementares, etc.

Não estamos afirmando que tais avaliações não possam ser feitas, o que deve ser problematizado é a forma como são feitas e o que se espera com o resultado. É essencial que as condições de saúde dos sujeitos sejam observadas, não só as relacionadas às questões físicas e fisiológicas (biológicas), mas também as relacionadas à dinâmica sociofamiliar e laboral, mas tradicionalmente essas avaliações só buscam dados antropométricos: peso corporal, altura, circunferência abdominal, da cintura e do quadril e dificilmente são incluídas questões mais gerais, relacionadas ao contexto de vida dos sujeitos.

Se houver a opção pela medição indireta de parâmetros fisiológicos, como a frequência cardíaca, o consumo de oxigênio ou da composição corporal, medidores associados à aptidão física e muito comumente extrapolados com sendo prova de que um sujeito é saudável, é necessário ter claro que os resultados provenientes não são a única forma de se avaliar os resultados das $\mathrm{PC}$ e AF quando relacionadas à saúde.

Utilizaremos uma situação hipotética para ilustrar: um senhor que acabou de perder um familiar com quem conviveu durante muitos anos, ao apresentar um estado de luto e chegar à Unidade Básica de Saúde ou no polo do Programa Academia da Saúde, ou no espaço público que os trabalhadores da $A B$ utilizam para desenvolver atividades comunitárias, iniciou a realização das PC e AF. A partir do acolhimento dos trabalhadores da saúde, dos usuários, do convívio e compartilhamento de experiências, seu quadro mudou e ele passou a encarar a vida de outra forma, claramente melhorando sua qualidade de vida. Se não forem medidos sistematicamente os parâmetros fisiológicos citados é possível afirmar que não houve melhora na sua saúde? Este é um exemplo entre tantos outros possíveis.

Além disso, os parâmetros citados não podem ser utilizados como algo fechado e definitivo. Um sujeito com tais medições dentro dos padrões de normalidade está inserido em um dado contexto, que fará com que outras opções saudáveis sejam mais ou menos possíveis. 
Assim, a partir do conhecimento das possibilidades dos usuários que realizarão as PC e AF, deve-se analisar a necessidade de realização de avaliações específicas.

Também é necessário conhecer as experiências, interesses, expectativas e disponibilidade desses sujeitos. Os trabalhadores e profissionais de saúde devem estar atentos à execução das PC e AF, saber como os sujeitos estão reagindo a elas, enfim, deve haver a troca de informações e conhecimentos sobre a atividade proposta e/ou realizada. A diversificação das modalidades das PC e AF é essencial para que o maior número de usuários possa e queira participar. Dada a diversidade regional brasileira, devem ser respeitadas as características culturais locais, mas nada impede de serem apresentadas novas atividades. É necessário saber o que os participantes acharam desta nova atividade e dar oportunidade para que essas opiniões permitam a ressignificação da prática. Em ambos os casos, as atividades devem considerar as necessidades dos usuários e os determinantes e condicionantes da saúde.

As atividades coletivas têm a potencialidade da formação de grupos heterogêneos, 0 que fará as pessoas vivenciarem as diferenças e perceber que elas não devem ser impeditivas e, sim, constituintes da diversidade que é inerente à condição humana. Além disso, o estabelecimento de um ambiente acolhedor no qual as diferenças sejam tratadas de maneira positiva tornará as atividades mais atrativas. É possível levantar e debater questões de gênero, étnico-raciais, ligadas aos ciclos de vida, a competitividade exacerbada, os padrões estéticos, doenças prevalentes, enfim, são muitas as possibilidades.

Destaca-se também que atualmente questões como beleza, estética, modismo e culto ao corpo recebem grande enfoque e as PC e AF são diretamente relacionadas a elas (BAPTISTA, 2013). Tais questões ganham contornos identitários (BAGRICHEVSKY, 2006, CASTIEL; VASCONCELLOS-SILVA, 2006), ou seja, uma determinada forma física corporal pode significar aceitação ou rejeição. A aparente dicotomia entre estética e saúde carece de abordagem na $A B$, elas podem andar juntas quando: se aceita o que é independentemente dos padrões de corpos hegemônicos, se entende que cada pessoa é única e o seu corpo é a sua forma de interagir com o mundo, ou podem afastar-se já que nem sempre um corpo "sarado" é um corpo saudável, isto a partir de opções do uso de substâncias ou comportamentos que possam comprometer seriamente a saúde de quem as usa ou faz, os esteroides anabolizantes e dietas "mirabolantes" são exemplos.

De forma geral, há o entendimento, muitas vezes implícito até no pensamento de trabalhadores e profissionais de saúde, de que características morfológicas como magreza ou corpos musculosos são exemplos de saúde, denotando a concepção que privilegia o biológico quando refere à saúde (CARVALHO, 2008). A priori, não há nenhum problema em querer mudar a aparência física, isso pode até significar melhorar a condição de saúde, por exemplo, se pensarmos na obesidade. Quando falamos sobre aparência física, isso também inclui a perda de peso que faz parte de um projeto ou plano terapêutico na doença citada. Contudo, a forma como isso será feito deve ser objeto de reflexão, problematização e ação. $O$ que se quer evitar é a busca de corpos baseados em modelos externos, muitas vezes inalcançáveis.

\section{CONSIDERAÇÕES}

Este ensaio tem um caráter reflexivo e propositivo, não é objetivo dele esgotar todas as possibilidades do tema, mas sim fazer um convite para a construção de uma gama maior 
de alternativas para as PC e AF como ferramentas de promoção da saúde e produção do cuidado na $A B$, buscando superar o foco exclusivo na prevenção das DCNTs, sem diminuir a importância dessas ações, em especial no atual quadro epidemiológico da população brasileira no qual essas doenças são umas das principais causas de morbimortalidade (BRASIL, PNS, 2014b).

Defende-se a oferta das $\mathrm{PC}$ e $\mathrm{AF}$ na $\mathrm{AB}$ num sentido mais amplo do que a compreensão delas apenas como fator de proteção contra as DCNTs, destacamos que a prevenção e tratamento dessas e outras doenças também são objetivos, mas não os únicos. Segundo Matiello Júnior et al. (2008), a lógica dos fatores de risco está impregnada em nossos saberes e agires cotidianos.

Faz-se necessário então a superação desta lógica, que não a exclua, mas que articule as perspectivas sociais, culturais, econômicas e políticas, além das biológicas, no processo saúde-doença e na realização das PC e AF como hábitos de vida. Assim, a aptidão e o condicionamento físico não são os objetivos exclusivos destas práticas na $A B$, a melhora destes é desejada, mas tal ocorrência deve ser complementar e processual.

As PC e AF possivelmente não contribuirão para qualificar o processo de trabalho das equipes de saúde, ampliar a resolutividade, entre outros objetivos da $A B$, caso resumam-se a uma atividade com fim em si mesma, por exemplo, uma aula de ginástica muito animada e cheia, mas que não tenha nenhum elemento de educação em saúde, ou seja, sejam realizados os movimentos corporais e em nenhum momento seja feita a relação deles com o processo de cuidado à saúde individual e coletiva por se acreditar que apenas a movimentação corporal e a medição e acompanhamento de determinados parâmetros fisiológicos bastam. As atividades que envolvem as PC e AF devem considerar sua sustentabilidade, planejando como os sujeitos terão sua autonomia aumentada em relação não só a elas.

Assim, na AB, a partir da utilização do conhecimento do território e da identificação de suas potencialidades, da criação e fortalecimento de vínculos, da continuidade e longitudinalidade do cuidado, da consideração de que diversos fatores como os sociais, econômicos, culturais e políticos influenciam as condições de vida e saúde de sujeitos e coletividades, é possível e mais factível conseguir mudanças efetivas nas condições de saúde individual e coletiva a partir da realização das PC e AF. E a partir daí apoiar as mudanças necessárias para o aumento da autonomia de sujeitos e coletividades no processo saúde-doença.

Para isso, o encontro é condição fundamental, destaca-se a convivência, a formação e fortalecimento de grupos sociais nos territórios ao criar e/ou aumentar o vínculo entre os sujeitos e destes com os trabalhadores e serviços de saúde como importantes objetivos. As PC e AF na $A B$ devem fazer parte de um contexto maior, o qual busca ampliar e garantir a integralidade do cuidado, logo, não têm fim em si mesmas. Devem trazer não só benefícios biológicos, mas também devem propiciar o aumento do círculo social de convivência, de compartilhamento de objetivos, aflições, soluções e respostas para as mais variadas situações que a vida apresenta.

Assim, estão inseridas no desafio que envolve trabalhadores e serviços de saúde da $A B$, qual seja, envolver e apoiar os sujeitos e coletividades, considerando os determinantes e condicionantes da saúde, com o objetivo de aumentar as opções para que estas façam aquelas que forem mais saudáveis. Para isso, é essencial que seja levada em consideração a abordagem integral do sujeito em seu contexto social, familiar e cultural, além do biológico. 
Buscar-se-á valorizar as experiências corporais dos sujeitos e as subjetividades criadas a partir daí, sem desconsiderar a perspectiva biológica e nem supervalorizá-la.

\section{REFERÊNCIAS}

ANDRADE, Douglas Roque. Políticas recentes de promoção da atividade física no Brasil. Corpoconsciência, Santo André, v. 15, n. 2, p. 2-6, 2011.

BAGRICHEVSKY, Marco et al. Apresentação. In: BAGRICHEVSKY, Marco (Org.). A saúde em debate na educação física. Blumenau: Nova Letra, 2006. v. 2, p. 7-15.

BAGRICHEVSKY, Marco; ESTEVÃO, Adriana. Perspectivas para a formação profissional em educação física: o SUS como horizonte de atuação. Arquivos em Movimento, Rio de Janeiro, v. 4, n. 1, p. 128-143, 2008.

BAPTISTA, Tadeu João Ribeiro. Corpo, estética, exercício e saúde coletiva. Praxia, Quirinópolis, v. 1 , n. 3, p. 4-24, 2013.

BRASIL. Ministério da Saúde. Avaliação de efetividade de programas de atividade física no Brasil. Brasília, 2011.

BRASIL. Ministério da Saúde. Curso de extensão em promoção da saúde para gestores do SUS com enfoque no Programa Academia da Saúde. Brasília, 2013.

BRASIL. Ministério da Saúde. Divulgação de dados sobre o Programa Academia da Saúde. Brasília, 2014a.

BRASIL. Ministério da Saúde. Glossário temático: promoção da saúde. Brasília, 2012a.

BRASIL. Ministério da Saúde. Instituto Brasileiro de Geografia e Estatística. Ministério do Planejamento, Orçamento e Gestão. Pesquisa nacional de saúde: percepção do estado de saúde, estilos de vida e doenças crônicas. Brasil, grandes regiões e unidades da federação. Rio de Janeiro, $2014 b$.

BRASIL. Ministério da Saúde. Política nacional de atenção básica. Brasília, 2012b.

CASTIEL, Luis David; VASCONCELLOS-SILVA, Paulo Roberto. A noção de 'estilo de vida' em promoção de saúde: um exercício crítico de sensibilidade epistemológica. In: BAGRICHEVSKY, Marco (Org.). A saúde em debate na educação física. Blumenau, Nova Letra, 2006. v. 2, p. 67-90.

CARVALHO, Fabio Fortunato Brasil de. Atividade Física na perspectiva crítica de Promoção da Saúde: por outra compreensão da educação física. Monografia (Especialização em Saúde Pública) Escola Nacional de Saúde Pública Sérgio Arouca, Fundação Oswaldo Cruz, Rio de Janeiro, 2008.

CARVALHO, Fabio Fortunato Brasil de. Análise crítica da carta brasileira de prevenção integrada na área da saúde na perspectiva da Educação Física através do enfoque radical de promoção da saúde. Saúde e Sociedade. São Paulo, v.18, n. 1, p. 227-236, 2009.

CARVALHO, Fabio Fortunato Brasil de. Educação Física e Saúde Coletiva: diálogo e aproximação. Corpus et Scientia, Rio de Janeiro, v.8, n. 3, p.109-126, 2012a.

CARVALHO, Fabio Fortunato Brasil de. A saúde na escola e a Promoção da Saúde: conhecendo a dinâmica intersetorial em Duque de Caxias e Nova Iguaçu - RJ. Dissertação (Mestrado em Saúde Pública) - Escola Nacional de Saúde Pública, Fundação Oswaldo Cruz, 2012b.

CARVALHO, Yara Maria de. Práticas corporais e comunidade: um projeto de educação física no Centro de Saúde Escola Samuel B. Pessoa (USP). In: FRAGA, Alex Branco,

WACHS, Felipe (Org.). Educação física e saúde coletiva: políticas de formação e perspectivas de intervenção. 2. ed. Porto Alegre: UFRGS Editora, 2007. p. 63-72. 
CRUZ, Danielle Keylla Alencar. Da promoção à prevenção: o processo de formulação da política nacional de promoção da saúde no período de 2003 a 2006. Dissertação (Mestrado em Ciências da Saúde) - Centro de Pesquisas Aggeu Magalhães, Fundação Oswaldo Cruz, Recife, 2010. Disponível em: <http://www.cpqam.fiocruz.br/bibpdf/2010cruz-dka.pdf>. Acesso em: 05 de maio de 2015.

DAMICO, José Geraldo Soares; KNUTH, Alan Goularte. O des(encontro) das práticas corporais e atividade física: hibridizações e borramentos no campo da saúde. Movimento, Porto Alegre, v. 20, n. 1, p. 329-350, 2014

FERLA, Antônio Alcindo et al. Educação Física e saúde coletiva: o que pode a ideia de integralidade na produção de mudanças no trabalho e na educação dos profissionais? In: FRAGA, Alex Branco, CARVALHO, Yara Maria, GOMES, Ivan Marcelo. (Org.). As práticas corporais no campo da saúde. São Paulo: Hucitec, 2013. p. 198-226.

FERREIRA, Marcos Santos; NAJAR, Alberto Lopes. Programas e campanhas de promoção da atividade física. Ciência \& Saúde Coletiva, Rio de Janeiro, v. 10, p. 207-219, 2005. Supl.

FRAGA, Alex Branco et al. As práticas corporais no campo da saúde. In: FRAGA, Alex Branco, CARVALHO, Yara Maria, GOMES, Ivan Marcelo. (Org.). As práticas corporais no campo da saúde. São Paulo: Hucitec, 2013. p. 7-20

FRAGA, Alex Branco. Promoção da vida ativa: nova ordem físico-sanitária na educação dos corpos contemporâneos. In: PALMA, Alexandre; ESTEVÃO, Adriana; DA ROS, Marco (Org.). A saúde em debate na educação física - volume 2. Blumenau: Nova Letra, 2006. p.105-120

LAZZAROTTI FILHO, Ari et al. O termo práticas corporais na literatura científica brasileira e sua repercussão no campo da Educação Física. Movimento, Porto Alegre, v. 16, n. 1, p. 11-29, jan./mar. 2010.

MALTA, Deborah Carvalho et al. Política Nacional de Promoção da Saúde, descrição da implementação do eixo atividade física e práticas corporais, 2006 a 2014. Revista Brasileira de Atividade Física e Saúde, Pelotas, v. 19, n. 3, p. 286-299, maio 2014.

MARTINEZ, Jéssica Félix Nicácio et al. Práticas corporais no SUS: tensões teóricas e práticas. In: FRAGA, Alex Branco, CARVALHO, Yara Maria, GOMES, Ivan Marcelo. (Org.). As práticas corporais no campo da saúde. São Paulo: Hucitec, 2013. p. 139-177.

MALTA, Deborah Carvalho et al. A promoção da saúde e da atividade física no Sistema único de Saúde. Revista Brasileira de Atividade Física e Saúde, Pelotas, v. 13, n. 1. p. 24-27, 2008.

MATIELLO JÚNIOR, Edgard et al. Superando riscos na atividade física relacionada à saúde. Movimento, Porto Alegre, v. 14, n. 1, p. 39-61, 2008.

MATTOS, Ruben Araújo de. Os sentidos da Integralidade: algumas reflexões acerca de valores que merecem ser defendidos. In: PINHEIRO, Roseni, MATTOS, Ruben Araújo de (Org.). Os sentidos da integralidade na atenção e no cuidado à saúde. 6. ed. Rio de Janeiro, IMS/UERJ - CEPESC ABRASCO, 2006. p. 39-64.

PALMA, Alexandre. Atividade física, processo saúde-doença e condições sócio-econômicas: uma revisão de literatura. Revista Paulista de Educação Física, São Paulo, 14(1):97-106, jan./jun. 2000.

PALMA, Alexandre; VILAÇA, Murilo Mariano. O sedentarismo da epidemiologia. Revista Brasileira de Ciências do Esporte, Campinas, v. 31, n. 2, p. 105-119, jan. 2010.

SILVA, Thaís Severino da; CARVALHO, Fabio Fortunato Brasil de. A promoção da saúde na atenção primária: possibilidades e desafios. In: KALINOWSKI, Carmen Elizabeth; FIGUEIREDO, Karla Crozeta; FONSECA, Rosa Maria Godoy Serpa da. (Org.). Programa de Atualização em Enfermagem: Atenção Primária e Saúde da Família: Ciclo 2. Porto Alegre: Artmed Panamericana, 2014. p. 71-92. 
TURA, Lurdes Maria Toazza et al. Atenção Primária em Saúde. In: SOUZA, Maria Fátima de; FRANCO, Marcos da Silveira; MENDONÇA, Ana Valéria Machado (Org.). Saúde da família nos municípios brasileiros: os reflexos dos 20 anos do espelho do futuro. Campinas: Saberes, 2014. p.178-205. 